\title{
Data Mapper: An operator for expressing one-to-many data transformations
}

\author{
Paulo Carreira ${ }^{1}$, Helena Galhardas ${ }^{2}$, João Pereira ${ }^{2}$, and Antónia Lopes ${ }^{1}$ \\ 1 Faculty of Sciences of the University of Lisbon, C6 - Piso 3, 1700 Lisboa, Portugal \\ paulo.carreira@oblog.pt ${ }^{\star \star}$, mal@di.fc.ul.pt \\ 2 INESC-ID and Instituto Superior Técnico, Avenida Prof. Cavaco Silva, Tagus \\ Park, 2780-990 Porto Salvo, Portugal \\ hig@inesc-id.pt, joao@inesc-id.pt
}

\begin{abstract}
Transforming data is a fundamental operation in application scenarios involving data integration, legacy data migration, data cleaning, and extract-transform-load processes. Data transformations are often implemented as relational queries that aim at leveraging the optimization capabilities of most RDBMSs. However, relational query languages like SQL are not expressive enough to specify an important class of data transformations that produce several output tuples for a single input tuple. This class of data transformations is required for solving the data heterogeneities that occur when source data represents an aggregation of target data.

In this paper, we propose and formally define the data mapper operator as an extension of the relational algebra to address one-to-many data transformations. We supply an algebraic rewriting technique that enables the optimization of data transformation expressions that combine filters expressed as standard relational operators with mappers. Furthermore, we identify the two main factors that influence the expected optimization gains.
\end{abstract}

\section{Introduction}

In general, data transformations aim at integrating data from multiple sources, migrating legacy data, cleaning data or performing ETL processes that support data warehousing. When putting in place such initiatives, data represented by a fixed source schema must be transformed into a fixed target data schema. In this context, we frequently face the problem of data heterogeneities, i.e., the use of different representations of the same data in source and target schemas [14, 11]. Several factors cause the existence of data heterogeneities, for example: (i) different units of measurement, (ii) different representations of compound data (e.g. multiple attributes representing day, month and year information $v s$ a single date attribute), or (iii) distinct representations of the same data domain (e.g. \{true, false\} vs $\{$ yes, no\} for boolean values). Another important source of data heterogeneities is the representation of data according to different aggregation

\footnotetext{
$\star \star$ The author's work was partially supported by OBLOG Consulting S.A.
} 
levels (e.g. hourly vs daily). When the source data represents an aggregation of the target data (e.g., yearly aggregated data in the source and monthly data in the target), the data transformation that has to take place needs to generate several output tuples for each input tuple. This class of data transformations will be henceforth designated as one-to-many data transformations.

Relational algebra (RA) expressions are often used to implement data transformations. In fact, simple data transformations can be naturally expressed as RA queries. Moreover, data often resides in RDBMSs and data transformations specified as relational expressions can take direct advantage of their optimization capabilities. However, due to the limitations in the expressive power of RA [1], relational queries are insufficient for expressing many interesting data transformations $[12,13]$.

The normalization theory underlying the relational model imposes the organization of data according to several relations in order to avoid duplication and inconsistency of information. Therefore, data retrieved from the database is mostly obtained by selecting, joining and unioning relations. Data transformation applications bring a new requirement to RA as their focus is no more limited to the idea of selecting information [1] but also involves the production of new data items. In particular, RA is not powerful enough to represent one-to-many data transformations.

In this paper, we propose to extend the RA with the mapper operator, which significantly increases its expressive power by representing one-to-many data transformations. Informally, a mapper is applied to an input relation and produces an output relation. It iterates over each input tuple and generates one or more output tuples, by applying a set of domain-specific functions. This way, it supports the dynamic creation of tuples based on a source tuple contents. This kind of operation also appears implicitly in most languages aiming at implementing schema and data transformations but, as far as we know, has never been properly handled as a first-class operator. Considering the mapper operator an extension to the RA brings new optimization opportunities as shown in [5].

The main contributions of this paper are the following:

- a formal definition of a new primitive operator, named data mapper, that allows expressing one-to-many data mappings;

- an algebraic rewriting technique for optimizing expressions involving mappers and filters expressed as relational operators;

- the identification of two important factors that influence the gains obtained when applying the proposed optimization.

The remainder of the paper is organized as follows. The formalization of the mapper is presented in Section 2. Section 3 presents the algebraic rewriting technique that enables the optimization of expressions involving the mapper operator. Practical evidence that shows the advantage of the optimization is presented in Section 4. Finally, related work is summarized in Section 5 and conclusions are presented in Section 6 . 


\subsection{Motivation}

As mentioned above, there is a considerable amount of data transformations that require one-to-many data mappings. In this section, we motivate the reader by presenting an example based on a real-world legacy-data migration scenario, that has been intentionally simplified for illustration purposes.

\begin{tabular}{rrrrrr}
\multicolumn{2}{c}{ Relation LOANS } & & \multicolumn{3}{c}{ Relation PAYMENTS } \\
\cline { 1 - 2 } \cline { 5 - 6 } ACCT & AM & & ACCTNO & AMOUNT & SEQNO \\
\hline 12 & 20.00 & & 0012 & 20.00 & 1 \\
3456 & 140.00 & & 3456 & 100.00 & 1 \\
901 & 250.00 & & 3456 & 40.00 & 2 \\
\cline { 1 - 3 } & & & 0901 & 100.00 & 1 \\
& & & 0901 & 100.00 & 2 \\
& & 0901 & 50.00 & 3 \\
\hline
\end{tabular}

Fig. 1. (a) On the left, the LOANS relation and, (b) on the right, the PAYMENTS relation.

Example 1. Consider the source relation LOANS[ACCT, AM] (represented in Figure 1) that stores the details of loans requested per account. Suppose LOANS data must be transformed into PAYMENTS[ACCTNO, AMOUNT, SEQNO], the target relation, according to the following requirements:

1. In the target relation, all the account numbers are left padded with zeroes. Thus, the attribute ACCTNO is obtained by (left) concatenating zeroes to the value of ACCT.

2. The target system does not support payment amounts greater than 100 . The attribute AMOUNT is obtained by breaking down the value of AM into multiple parcels with a maximum value of 100 , in such a way that the sum of amounts for the same ACCTNO is equal to the source amount for the same account. Furthermore, the target field SEQNO is a sequence number for the parcel. This sequence number starts at 1 for each sequence of parcels of a given account.

The implementation of data transformations similar to those requested for producing the target relation PAYMENTS of Example 1 is challenging, since solutions to the problem involve the dynamic creation of tuples based on the value of attribute AM.

\section{The mapper operator}

We start by introducing some preliminary notation. Let $R\left(A_{1}, \ldots, A_{n}\right)$ be a relation schema. The domain of the relation schema $R$ is represented as $\operatorname{Dom}(A)$, where $A$ represents the relation schema composed by the attributes $A_{1}, \ldots, A_{n}$. A relation instance (or relation, for short) of $R(A)$ is written as $r(A)$ or simply $r$. Each element $t$ of $r$ is called a tuple or $r$-tuple and can be regarded as a function that associates a value of $\operatorname{Dom}\left(A_{i}\right)$ to each $A_{i} \in A$; we denote this value by 
$t\left[A_{i}\right]$. Given a set of distinct attributes $B=\left\{B_{1}, \ldots, B_{k}\right\}$ where each $B_{i} \in A$, we denote by $t[B]$, the tuple $\left\langle t\left[B_{1}\right], \ldots, t\left[B_{k}\right]\right\rangle$. We assume two fixed relation schemas $S\left(X_{1}, \ldots, X_{n}\right)$ and $T\left(Y_{1}, \ldots, Y_{m}\right)$. We refer to $S$ and $T$ as the source and the target relation schemas, respectively.

A mapper is a unary operator $\mu_{F}$ that takes a relation instance of the source relation schema as input and produces a relation instance of the target relation schema as output. The mapper operator is parameterized by a set $F$ of special functions, which we designate as mapper functions.

Roughly speaking, each mapper function allows one to express a part of the envisaged data transformation, focused on one or more attributes of the target schema. Although the idea is to apply mapper functions to tuples of a source relation instance, it may happen that some of the attributes of the source schema are irrelevant for the envisaged data transformation. The explicit identification of the attributes that are considered relevant is then an important part of a mapper function. Mapper functions are formally defined as follows.

Definition 1. Let $A$ be a non-empty list of distinct attributes in $Y_{1}, \ldots, Y_{m}$. An A-mapper function for transforming the data of $S$ into $T$ consists of a non-empty list of distinct attributes $B$ in $X_{1}, \ldots, X_{n}$ and a computable function $f_{A}: \operatorname{Dom}(B) \rightarrow \mathcal{P}(\operatorname{Dom}(A))$.

Let $t$ be tuple of a relation instance of the source schema. We define $f_{A}(t)$ to be the application of the underlying function $f_{A}$ to the tuple $t$, i.e., $f_{A}(t[B])$.

We shall freely use $f_{A}$ to denote both the mapper function and the function itself. In this way, mapper functions describe how a specific part of the target data can be obtained from the source data. The intuition is that each mapper function establishes how the values of a group of attributes of the target schema can be obtained from the attributes of the source schema. Another key point is that, when applied to a tuple, a mapper function can produce a set of values of $\operatorname{Dom}\left(f_{A}\right)$, rather than a single value.

As mentioned before, a mapper operator is parameterized by a set of mapper functions. This set is said to be proper for transforming the data from the source to the target schemas if it specifies, in a unique way, how the values of every attribute of the target schema are produced.

Definition 2. A set $F=\left\{f_{A_{1}}, \ldots, f_{A_{k}}\right\}$ of mapper functions is said to be proper (for transforming the data of $S$ into $T$ ) iff every attribute $Y_{i}$ of the target relation schema is an element of exactly one of the $A_{j}$ lists, for $1 \leq j \leq k$.

The mapper operator $\mu_{F}$ puts together the data transformations of the input relation defined by the mapper functions in $F$. Given a tuple $s$ of the input relation, $\mu_{F}(s)$ consists of the tuples $t$ in $\operatorname{Dom}(Y)$ such that, for every $i$, to the attributes in $A_{i}$, associate the values given by $f_{A_{i}}(s)$. Formally, the mapper operator is defined as follows.

Definition 3. Given a relation $s(X)$ and a proper set of mapper functions $F=\left\{f_{A_{1}}, \ldots, f_{A_{k}}\right\}$, the mapper of $s$ with respect to $F$, denoted by $\mu_{F}(s)$, is the relation instance of the target relation schema defined by 


$$
\mu_{F}(s) \stackrel{\text { def }}{=}\left\{t \in \operatorname{Dom}(Y) \mid \exists u \in \text { s s.t. } t\left[A_{i}\right] \in f_{A_{i}}(u), \forall 1 \leq i \leq k\right\}
$$

The semantics of the mapper operator can be given also as a Cartesian product of the results of each mapper function. Definition 3 is more abstract than the definition in terms of Cartesian product operations in the sense that it does not impose any particular ordering on the attributes. For further details about the properties and expressiveness of the mapper operator, please refer to [5]. In order to illustrate this new operator, we revisit Example 1.

Example 2. The requirements presented in Example 1 can be described by the mapper $\mu_{\text {acct,amt }}$, where acct is an ACCT-mapper function that returns a singleton with the account number ACCT properly left padded with zeroes and amt is the [AMOUNT, SEQNO]-mapper function s.t., $\operatorname{amt}(\mathrm{am})$ is given by

$\{(100, i) \mid 1 \leq i \leq(a m / 100)\} \cup\{(a m \% 100,(a m / 100)+1) \quad \mid a m \% 100 \neq 0\}$ where we have used / and \% to represent the integer division and modulus operations, respectively.

For instance, if $t$ is the source tuple $(901,250.00)$, the result of evaluating $\operatorname{amt}(t)$ is the set $\{(100,1),(100,2),(50,3)\}$. Given a source relation $s$ including $t$, the result of the expression $\mu_{\text {acct }, a m t}(s)$ is a relation that contains the set of tuples $\left\{\left\langle{ }^{\prime} 0901^{\prime}, 100,1\right\rangle,\left\langle^{\prime} 0901^{\prime}, 100,2\right\rangle,\left\langle{ }^{\prime} 0901^{\prime}, 50,3\right\rangle\right\}$.

\section{Optimization of sequences of filters and mappers}

In this section, we present a logical optimization technique that comprises two algebraic rewriting rules for optimizing expressions that combine filters expressed as relational selection operators with mappers. The first rule presented alleviates the cost of performing the Cartesian product operations that are used to implement the mapper operator. The second rule avoids superfluous function evaluations by pushing selections to the sources, thus reducing the number of tuples fed to the mapper as early as possible. Each rule is presented as an equivalence between terms. This equivalence is complemented by a text that describes the semantic pre-conditions that guarantee its correctness.

\subsection{Pushing selections to mapper functions}

When applying a selection to a mapper we can take advantage of the mapper semantics to introduce an important optimization. Given a selection $\sigma_{C_{A_{i}}}$ applied to a mapper $\mu_{f_{A_{1}}, \ldots, f_{A_{k}}}$, this optimization consists of pushing the selection $\sigma_{C_{A_{i}}}$, where $C_{A_{i}}$ is a condition on the attributes produced by some mapper function $f_{A_{i}}$, directly to the output of the mapper function.

RULE 1: Let $F=\left\{f_{A_{1}}, \ldots, f_{A_{k}}\right\}$ be a set of multi-valued mapper functions, proper for transforming $S(X)$ into $T(Y)$. Consider a condition $C_{A_{i}}$ dependent on a list of attributes $A_{i}$ such that $f_{A_{i}} \in F$. Then,

$$
\begin{aligned}
& \sigma_{C_{A_{i}}}\left(\mu_{F}(s)\right)=\mu_{F \backslash\left\{f_{A_{i}}\right\} \cup\left\{\sigma_{C_{A_{i}}} \circ f_{A_{i}}\right\}}(s) \\
& \text { where }\left(\sigma_{C_{A_{i}}} \circ f_{A_{i}}\right)(t)=\left\{f_{A_{i}}(t) \mid C_{A_{i}}(t)\right\} .
\end{aligned}
$$


Note that when $C_{A_{i}}(t)$ does not hold, the function $\sigma_{C_{A_{i}}}\left(f_{A_{i}}\right)(t)$ returns the empty set. Whenever a mapper function $f_{A_{i}}$ applied to a tuple $t$ returns an empty set, the result of the mapper will also be an empty set. Hence, we may skip the evaluation of all mapper functions $f_{A_{j}}$, such that $j \neq i$. Physical execution algorithms for the mapper operator can take advantage of this optimization by evaluating $f_{A_{i}}$ before any other mapper function ${ }^{3}$.

\subsection{Pushing selections through mappers}

An alternative way of rewriting expressions of the form $\sigma_{C}\left(\mu_{F}(s)\right)$ consists of replacing the attributes that occur in the condition $C$ with the mapper functions that compute them. Suppose that, in the selection condition $C$, attribute $A$ is produced by the mapper function $f_{A}$. By replacing the attribute $A$ with the mapper function $f_{A}$ in condition $C$ we obtain an equivalent condition.

In order to formalize this notion, we first need to introduce some notation. Let $F=\left\{f_{A_{1}}, \ldots, f_{A_{k}}\right\}$ be a set of mapper functions proper for transforming $S(X)$ into $T(Y)$. The function resulting from the restriction of $f_{A_{i}}$ to an attribute $Y_{j} \in A_{i}$ is denoted by $f_{A_{i}} \downarrow Y_{j}$. Moreover, given an attribute $Y_{j} \in Y, F \downarrow Y_{j}$ represents the function $f_{A_{i}} \downarrow Y_{j}$ s.t. $Y_{j} \in A_{i}$. Note that, because $F$ is a proper set of mapper functions, the function $F \downarrow Y_{j}$ exists and is unique.

RULE 2: Let $F=\left\{f_{A_{1}}, \ldots, f_{A_{k}}\right\}$ be a set of single-valued mapper functions, proper for transforming $S(X)$ into $T(Y)$. Let $B=B_{1} \cdot \ldots \cdot B_{k}$ be a list of attributes in $Y$ and $s$ a relation instance of $S(X)$. Then,

$$
\sigma_{C_{B}}\left(\mu_{F}(s)\right)=\mu_{F}\left(\sigma_{C\left[B_{1}, \ldots, B_{k} \leftarrow F \downarrow B_{1}, \ldots, F \downarrow B_{k}\right]}(s)\right)
$$

where $C_{B}$ means that $C$ depends on the attributes of $B$, and the condition that results from replacing every occurrence of each $B_{i}$ by $E_{i}$ is represented as $C\left[B_{1}, \ldots, B_{n} \leftarrow E_{1}, \ldots, E_{n}\right]$.

This rule replaces each attribute $B_{i}$ in the condition $C$ by the expression that describes how its values are obtained.

\section{Practical Validation}

In order to validate the optimizations proposed in Section 3, we have implemented the mapper operator and conducted a number of experiments contrasting expressions consisting of mappers and selections with their optimized equivalents. Due to space limitations we only focus on the optimization obtained by applying Rule 1.

We have identified the mapper function fanout and the predicate selectivity [16], as two important factors that influence the gain obtained when applying

\footnotetext{
3 This optimization can be generalized to first evaluate those functions with higher probability of yielding an empty set. Fundamentally, this is the same as finding the optimal predicate ordering addressed in [10].
} 
the proposed optimization. Similarly to [6], we designate the average cardinality of the output values produced by a mapper function for each input tuple as function fanout. Our experiments only address the influence of the two above mentioned factors by employing cheap functions. Other factors that influence the mapper execution cost are the $\mathrm{I} / \mathrm{O}$ cost and the function evaluation $\operatorname{cost}^{4}$.

The experiments apply a mapper $\mu_{f_{1}, f_{2}, f_{3}, f_{4}}$ to synthetic data where, unless otherwise stated, each mapper function has a fanout factor of 2.0. In all experiments, we test the original expression $\sigma_{p_{i}}\left(\mu_{f_{1}, f_{2}, f_{3}, f_{4}}(r)\right)$ and the optimized expression $\mu_{f_{1}, \sigma_{p_{i}} \circ f_{2}, f_{3}, f_{4}}(r)$, where predicate $p_{i}$ has some predefined selectivity and $r$ is an input relation with a predefined size. Each experiment measures total work, i.e., the sum of the time taken to read the input tuples, to compute the output tuples, and to materialize them.
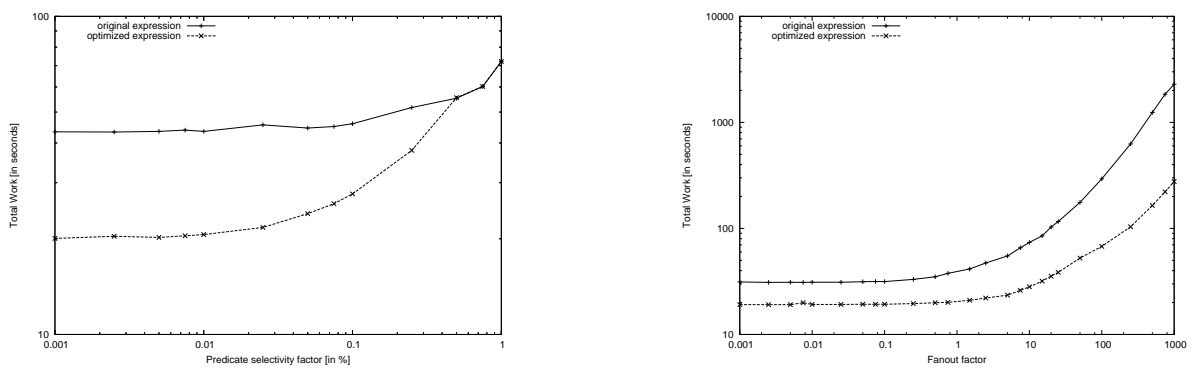

Fig. 2. Evolution of total work for the original and optimized expressions with 1 million tuples. (a) On the left, the effect of increasing selectivity factors for a mapper with four functions with a fanout factor of 2.0 (b) On the right, the effect of increasing mapper function fanout factors with a predicate selectivity fixed to $2.5 \%$.

The influence of predicate selectivity To understand the effect of the predicate selectivity, a set of experiments was carried out using a different $p_{i}$ predicate with selectivity factor ranging from $0.1 \%$ to $100 \%$. The tests were executed over an input relation with 1 million input tuples. Figure 2a shows the evolution of the total work for different selectivities.

As expected, the highest gains brought by the optimization were obtained for smaller selectivity factors. More concretely, for a selectivity of $0.1 \%$, the optimized expression was 2.16 times faster than the original one. As the selectivity factor decreases, more results are filtered out from function $f_{2}$ and, therefore, the cost of computing the Cartesian product involved in the mapper is lower. As the selectivity tends to $100 \%$, the gain drops since the results filtered out from $f_{2}$ tend to $0 \%$. Nevertheless, there is still, albeit small, a gain due to the reduction on the number of predicate evaluations (recall that each function has a fanout of 2). This gain is small since the cost of a predicate evaluation is, in our experiments, low.

\footnotetext{
${ }^{4}$ We do not take into account these factors in our experiments, since Rule 1 is not able to optimize them.
} 
The influence of function fanout To understand the effects of the function fanout on the optimization proposed, we tracked the evolution of total work for the original and optimized expressions when the fanout factor varies. Function $f_{2}$ was replaced by a function that guarantees a predefined fanout factor ranging from 0.001 (unusually small) to 1000 . To isolate the effect of the fanout, the fanout factors of the remaining functions were set to 1.0 and the selectivity of the predicate was kept constant at 2.25\%. The results are depicted in Figure 2b.

For small values of fanout, the gain brought by the optimization remains mostly constant (an improvement of $\approx 61 \%$ ). This is due to the fact that for low values of fanout, the cost of performing the Cartesian product is lower than the combined costs of $\mathrm{I} / \mathrm{O}$ and evaluation of the mapper functions. Notice that the cost of the Cartesian product increases with the fanout, since the higher the fanout, the more tuples have to be produced by the Cartesian product for each input tuple. Thus, for high fanout values, the cost of performing the Cartesian product becomes the dominant factor. Hence, the gain of the optimization increases with the fanout factor since our optimization reduces the cost of the Cartesian product.

\section{Related work}

Data transformation is an old problem and the idea of using a query language to specify such transformations has been proposed back in the 1970's with two prototypes, Convert [17] and Express [18], both aiming at data conversion. More recently, three efforts, Potter's Wheel [15], Ajax [7] and Data Fusion [3], have proposed operators for data transformation and cleaning purposes.

Potter's Wheel fold operator is capable of producing several output tuples for each input tuple. The main difference w.r.t. the mapper operator lies in the number of output tuples generated. In the case of the fold operator, the number of output tuples is bound to the number of columns of the input relation, while the mapper operator may generate an arbitrary number of output tuples.

The semantics of the Ajax map operator represents exactly a one-to-many mapping, but it has not been proposed as an extension of the relational algebra. Consequently, the issue of semantic optimization, as we propose in this paper, has not been addressed for the Ajax map. Data Fusion implements the semantics of the mapper operator as it is presented here. However, the current version of Data Fusion is not supported by an extended relational algebra as we propose.

Our decision of adopting database technology as a basis for data transformation is not completely revolutionary (see, e.g., [9,2]). Several RDBMSs, like Microsoft SQL Server, already include additional software packages specific for ETL tasks. However, to the best of our knowledge, none of these extensions is supported by the corresponding theoretical background in terms of existing database theory. Therefore, the capabilities of relational engines, for example, in terms of optimization opportunities are not fully exploited for ETL tasks.

Recently, [19] has proposed a rigorous approach to the problem of optimizing an ETL process defined as a workflow of data transformation activities. The 
authors model the ETL optimization problem as a global state-space search problem. In our approach, we use local optimization, since an ETL transformation program must be represented by a set of extended relational algebra expressions to be optimized one at a time.

A preliminary version of this work [4] has presented the idea of performing the logical optimization of queries involving mappers and standard relational operators. However, the formal aspects concerning the mapper operator and the optimization rules were not detailed. In [5], we analyzed the optimization of expressions that combine mappers with other relational algebra operators and presented the formal correctness proofs for the rules.

\section{Conclusions and future work}

In this paper, we have addressed the problem of specifying one-to-many data transformations that are frequently required in data integration, data cleaning, legacy-data migration, and ETL scenarios. Practical evidence gathered from the Ajax [8] data cleaning prototype and from Data Fusion [3] legacy-data migration tool, which has been used commercially in large legacy-data migration projects, corroborates the need of supporting data transformations that require one-tomany mappings. Since one-to-many data transformations are not expressible through standard RA queries, we proposed the mapper operator. This new operator allow us to naturally express one-to-many data transformations, while extending the expressive power of RA at the same time. We show in [5] that RA extended with the mapper operator is more expressive than standard RA.

We presented a simple formal semantics for the mapper operator that can be implemented using Cartesian product operations. We then introduced two provenly correct algebraic rewriting rules that aim at optimizing queries that combine standard relational filters and data transformations encoded as mappers. To assess the effectiveness of our approach, we have implemented the mapper operator and conducted a set of experiments for observing the behavior of the rewriting rule that consists of pushing selection conditions to the output of mapper functions. Our experiments indicate that substantial performance improvements are obtained by employing the proposed rewriting, even in the presence of cheap functions. Furthermore, we were able to isolate two important factors, the predicate selectivity and the mapper function fanout, which strongly influence the performance gains obtained.

Currently, we are developing and experimenting different physical execution algorithms for the mapper operator. This way, we intend to complement the logical optimization technique presented with physical optimizations that can be integrated into an RDBMS optimizer. We strongly believe that this solution will definitely contribute to the application of the current relational database technology for enhancing the performance of data transformation engines. 


\section{References}

1. A. V. Aho and J. D. Ullman. Universality of data retrieval languages. In Proc. of the 6th ACM SIGACT-SIGPLAN Symposium on Principles of Programming Languages, pages 110-119. ACM Press, 1979.

2. P. A. Bernstein and E. Rahm. Data wharehouse scenarios for model management. In Int'l Conf. on Conceptual Modeling / the Entity Relationship Approach, 2000.

3. P. Carreira and H. Galhardas. Efficient development of data migration transformations. In ACM SIGMOD Int'l Conf. on the Managment of Data, June 2004.

4. P. Carreira and H. Galhardas. Execution of Data Mappers. In Int'l Workshop on Information Quality in Information Systems. ACM, June 2004.

5. P. Carreira, H. Galhardas, A. Lopes, and J. Pereira. Extending the relational algebra with the Mapper operator. DI/FCUL TR 05-2, Department of Informatics, University of Lisbon, January 2005. Available at the url http://www.di.fc.ul.pt/tech-reports.

6. S. Chaudhuri and K. Shim. Query optimization in the presence of foreign functions. In Proc. of the Int'l Conf. on Very Large Data Bases (VLDB'93), 1993.

7. H. Galhardas, D. Florescu, D. Shasha, and E. Simon. Ajax: An extensible data cleaning tool. ACM SIGMOD Int'l Conf. on Management of Data, 2(29), 2000.

8. H. Galhardas, D. Florescu, D. Shasha, E. Simon, and C.-A. Saita. Declarative Data Cleaning: Language, Model, and Algorithms. In Proc. of the Int'l Conf. on Very Large Data Bases (VLDB'01), Rome, Italy, September 2001.

9. L. Haas, R. Miller, B. Niswonger, M. T. Roth, P.M. Scwarz, and E. L. Wimmers. Transforming heterogeneous data with database middleware: Beyond integration. Special Issue on Data Transformations. IEEE Data Eng. Bulletin, 22(1), 1999.

10. J. M. Hellerstein. Optimization techniques for queries with expensive methods. ACM Transactions on Database Systems, 22(2):113-157, June 1998.

11. W. Kim, B.-J. Choi, E.-K. Hong, S.-K. Kim, and D. Lee. A taxonomy of dirty data. Data Mining and Knowledge Discovery, 7(1):81-99, January 2003.

12. L. V. S. Lakshmanan, F. Sadri, and I. N. Subramanian. SchemaSQL - A Language for Querying and Restructuring Database Systems. In Proc. Int'l Conf. on Very Large Databases (VLDB'96), pages 239-250, Bombay, India, September 1996.

13. R. J. Miller. Using Schematically Heterogeneous Structures. Proc. of ACM SIGMOD Int'l Conf. on the Managment of Data, 2(22):189-200, June 1998.

14. E. Rahm and H.-H. Do. Data Cleaning: Problems and current approaches. IEEE Bulletin of the Technical Comittee on Data Engineering, 24(4), 2000.

15. V. Raman and J. M. Hellerstein. Potter's Wheel: An Interactive Data Cleaning System. In Proc. of the Int'l Conf. on Very Large Data Bases (VLDB'01), 2001.

16. P. G. Selinger, M. M. Astrahan, D. D. Chamberlin, R. A. Lorie, and T. G. Price. Access path selection in a relational database management system. In ACM SIGMOD Int'l Conf. on the Managment of Data, 1979.

17. N. C. Shu, B. C. Housel, and V. Y. Lum. CONVERT: A High Level Translation Definition Language for Data Conversion. Communications of the ACM, 18(10):557-567, October 1975.

18. N. C. Shu, B. C. Housel, R. W. Taylor, S. P. Ghosh, and V. Y. Lum. EXPRESS: A Data EXtraction, Processing and REStructuring System. ACM Transactions on Database Systems, 2(2):134-174, June 1977.

19. A. Simitsis, P. Vassiliadis, and T. K. Sellis. Optimizing ETL processes in data warehouses. In Proc. of the 21st Int'l Conf. on Data Engineering (ICDE), 2005. 